Margarida Mendes Lopes · Rita Pardini

\title{
Numerical Campedelli surfaces with fundamental group of order 9
}

Received May 9, 2006 and in revised form November 20, 2006

\begin{abstract}
We give explicit constructions of all the numerical Campedelli surfaces, i.e. the minimal surfaces of general type with $K^{2}=2$ and $p_{g}=0$, whose fundamental group has order 9 . There are three families, one with $\pi_{1}^{\text {alg }}=\mathbb{Z}_{9}$ and two with $\pi_{1}^{\text {alg }}=\mathbb{Z}_{3}^{2}$.

We also determine the base locus of the bicanonical system of these surfaces. It turns out that for the surfaces with $\pi_{1}^{\text {alg }}=\mathbb{Z}_{9}$ and for one of the families of surfaces with $\pi_{1}^{\text {alg }}=\mathbb{Z}_{3}^{2}$ the base locus consists of two points. To our knowlegde, these are the only known examples of surfaces of general type with $K^{2}>1$ whose bicanonical system has base points.
\end{abstract}

Keywords. Campedelli surface, surface with $p_{g}=0$, fundamental group, torsion

\section{Introduction}

A numerical Campedelli surface is a minimal surface of general type with $K^{2}=2$ and $p_{g}=0$. It is known (cf. [BPHV] Chap. VII.10]) that the algebraic fundamental group $\pi_{1}^{\text {alg }}$ of such a surface is finite, of order at most 9 . In this paper, we construct explicitly all the numerical Campedelli surfaces with $\left|\pi_{1}^{\mathrm{alg}}\right|=9$.

For this, given a surface $S$ as above, we consider its universal cover $Y \rightarrow S$. Assume for simplicity that $K_{S}$ is ample. Then by results of Konno ([Ko] $)$ the canonical map of $Y$ is an isomorphism and $Y$ is the intersection of a normal threefold $W \subset \mathbb{P}^{7}$ of degree 5 or 6 with a cubic hypersurface. Using Fujita's classification of these threefolds ([Fu1Fu3]), we prove that in our situation $W$ actually has degree 6 and that there are three possibilities for $W$, each giving rise to a family of numerical Campedelli surfaces with algebraic fundamental group of order 9 (Theorem 3.1 .

More precisely (cf. §3), there is an irreducible family of dimension 6 of numerical Campedelli surfaces with $\pi_{1}^{\text {alg }}=\mathbb{Z}_{9}$, and two irreducible families, of dimension respectively 7 and 6 , of numerical Campedelli surfaces with $\pi_{1}^{\text {alg }}=\mathbb{Z}_{3}^{2}$. Recall that the expected

M. Mendes Lopes: Departamento de Matemática, Instituto Superior Técnico, Universidade Técnica de Lisboa, Av. Rovisco Pais, 1049-001 Lisboa, Portugal; e-mail: mmlopes@math.ist.utl.pt

R. Pardini: Dipartimento di Matematica, Università di Pisa, Largo B. Pontecorvo, 5, 56127 Pisa, Italy; e-mail: pardini@dm.unipi.it

Mathematics Subject Classification (2000): 14J29 
number of moduli of a numerical Campedelli surface is 6; as far as we know, these are the only known examples in which the dimension of the moduli space is greater than expected.

Furthermore we prove (Theorem 4.2) that the moduli space of these surfaces has exactly two connected components, both irreducible, corresponding to $\pi_{1}^{\text {alg }}=\mathbb{Z}_{9}$ and $\pi_{1}^{\text {alg }}=\mathbb{Z}_{3}^{2}$. We also show that for all these surfaces $\pi_{1}^{\text {alg }}$ coincides with the topological fundamental group (Theorem 4.3).

Moreover, we determine the base locus $\Gamma$ of the bicanonical system $\left|2 K_{S}\right|$ (Theorem 4.4). If $\pi_{1}^{\text {alg }}(S)=\mathbb{Z}_{9}$, then $\Gamma$ consists of two points. If $\pi_{1}^{\text {alg }}(S)=\mathbb{Z}_{3}^{2}$, then $\Gamma$ is empty in general but there is a codimension 1 subvariety of the moduli space where $\Gamma$ consists of two points. This fact is quite surprising. Indeed, excluding the case $K^{2}=1$, $p_{g}=0$ in which $\left|2 K_{S}\right|$ is a pencil, the bicanonical map of a minimal surface of general type is generically finite ([Xi2] ) and, by the work of various authors (cf. [Ci]), it is known to be a morphism if either $p_{g}>0$ or $K^{2}>4$. To our knowledge, the examples presented here are the only known surfaces of general type with $K^{2}>1$ whose bicanonical map is not a morphism.

The paper is organized as follows. In $\S 2$ we establish some preliminaries. In $\S 3$ we state the classification theorem (whose proof will be given in \$5) and describe in detail the three families of Campedelli surfaces with $\pi_{1}^{\text {alg }}$ of order 9 . In $\$ 4$ we study the moduli and the bicanonical system of such surfaces and finally in $\$ 5$ we prove the classification theorem.

Notation. We work over the complex numbers. All varieties are projective algebraic. All the notation we use is standard in algebraic geometry. We just recall the definition of the numerical invariants of a smooth surface $S$ : the self-intersection number $K_{S}^{2}$ of the canonical divisor $K_{S}$, the geometric genus $p_{g}(S):=h^{0}\left(K_{S}\right)=h^{2}\left(\mathcal{O}_{S}\right)$, the irregularity $q(S):=h^{0}\left(\Omega_{S}^{1}\right)=h^{1}\left(\mathcal{O}_{S}\right)$ and the holomorphic Euler characteristic $\chi(S):=1+$ $p_{g}(S)-q(S)$.

\section{Set-up and preliminaries}

Here we establish the notation and recall or prove some facts that we use throughout the paper.

Let $S$ denote a numerical Campedelli surface, i.e. a minimal projective surface of general type with $K_{S}^{2}=2$ and $p_{g}(S)=0$ (and so $q(S)=0$ ), and let $G$ denote the algebraic fundamental group of $S$.

Proposition 2.1 ([|[Re2], cf. [MP]). The group $G$ is finite, of order $\leq 9$.

We assume from now on that $G$ has order 9 and we consider the corresponding étale $G$-cover $\pi: Y \rightarrow S$.

Proposition 2.2. (i) $Y$ is a smooth minimal surface of general type with $K_{Y}^{2}=18$, $p_{g}(Y)=8, q(Y)=0$, hence $K_{Y}^{2}=3 p_{g}(Y)-6$;

(ii) the canonical map $\varphi: Y \rightarrow \mathbb{P}^{7}$ is birational onto its image $V$. 
Proof. Since $\pi$ is étale, $Y$ is smooth and $K_{Y}=\pi^{*} K_{S}$ is nef and big. Hence $Y$ is minimal of general type and $K_{Y}^{2}=9 K_{S}^{2}=18, \chi(Y)=9 \chi(S)=9$. By Proposition 2.1. we have $\pi_{1}^{\text {alg }}(Y)=0$ and thus, in particular, $q(Y)=0$. It follows that $p_{g}(Y)=8$ and so $K_{Y}^{2}=3 p_{g}(Y)-6$.

Suppose that the canonical map $\varphi$ of $Y$ is not birational. Then, by [Ko], $\varphi$ is generically finite of degree 2 and its image is a ruled surface, which is rational by the regularity of $Y$. But then, by [Be, Cor. 5.8] (cf. [MP, Proposition 4.1]), $G=\mathbb{Z}_{2}^{r}$, contradicting the assumption that $G$ has order 9 .

Conversely, one has:

Proposition 2.3. Let $V$ be a surface with canonical singularities and ample $K_{V}$, and with $K_{V}^{2}=18, p_{g}(V)=8, q(V)=0$; let $G$ be a group of order 9 that acts freely on $V$. Then the quotient surface $S:=V / G$ is the canonical model of a numerical Campedelli surface with $\pi_{1}^{\text {alg }}=G$.

Proof. Since the quotient map $p: V \rightarrow S$ is étale, $S$ has canonical singularities and $K_{V}=$ $p^{*} K_{S}$. Since $K_{V}$ is ample, $K_{S}$ is also ample, and therefore $S$ is the canonical model of a surface of general type. Since $p$ is étale, we have $K_{S}^{2}=K_{V}^{2} / 9=2, \chi(S)=\chi(V) / 9=1$. In addition, $q(S)=0$ since $q(V)=0$, and thus $p_{g}(S)=0$. Let $S^{\prime} \rightarrow S$ and $Y \rightarrow V$ be the minimal resolutions. The surface $S^{\prime}$ is a numerical Campedelli surface and the map $p: V \rightarrow S$ induces an étale map $p^{\prime}: Y \rightarrow S^{\prime}$ with Galois group $G$. Therefore there is a surjection $\pi_{1}^{\text {alg }}\left(S^{\prime}\right) \rightarrow G$. By Proposition 2.1 this is actually an isomorphism.

The next result is needed in $\S 3$ to explain the choices of linearization in the construction of the examples. It is well known to experts (cf. [ [Re2]), but we recall it here for lack of a published reference.

Proposition 2.4. The representation of $G$ on $H^{0}\left(Y, K_{Y}\right)$ decomposes as the direct sum of the eight nontrivial characters of $G$.

Proof. Let $\chi \in G^{*}$ be a character of $G$ and $V_{\chi} \subset H^{0}\left(Y, K_{Y}\right)$ the corresponding eigenspace. There is a natural isomorphism $G^{*} \rightarrow$ Tors $S$, the torsion subgroup of Pic $S$, which induces a natural identification $V_{\chi} \cong H^{0}\left(S, K_{S}+\eta_{\chi}\right)$, where $\eta_{\chi}$ is the line bundle associated to $\chi \in G^{*}$. Notice that $V_{1}=\{0\}$, since $p_{g}(S)=0$. By Proposition 2.1. there is no irregular étale cover of $S$, hence for every $\eta \in$ Tors $S$ we have $h^{1}(S, \eta)=0$. Since $\chi(S)=1$, Serre duality gives $h^{0}\left(S, K_{S}+\eta\right)=1$ for every $\eta \in$ Tors $S \backslash\{0\}$, i.e. $\operatorname{dim} V_{\chi}=1$ for every $\chi \in G^{*} \backslash\{0\}$.

\section{The classification}

The main result of this paper, which will be proven in $\$ 5$, is the following classification theorem:

Theorem 3.1. Let $S$ be a numerical Campedelli surface with $\pi_{1}^{\mathrm{alg}}(S)$ of order 9 . Then:

(i) if $\pi_{1}^{\mathrm{alg}}(S) \cong \mathbb{Z}_{9}$, then $S$ is a surface of type $A$ (cf. $\$ 3.1$;

(ii) if $\pi_{1}^{\operatorname{alg}}(S) \cong \mathbb{Z}_{3}^{2}$, then $S$ is a surface of type $B 1$ or $B 2$ (cf. \$3.2. $\$ 3.3$ ). 
In this section we describe in detail the three families of Campedelli surfaces with $\pi_{1}^{\text {alg }}$ of order 9 (i.e., type A, type B1, type B2). The notation is consistent with the previous section.

The examples are obtained as quotients of surfaces $Y$ with $K_{Y}^{2}=18, p_{g}(Y)=8$ and $q(Y)=0$ by a group $G$ of order 9 acting freely.

In each case $Y$ is the minimal resolution of a surface $V$ of $\mathbb{P}^{7}$ with canonical singularities, obtained as the intersection of a normal threefold $W \subset \mathbb{P}^{7}$ of degree 6 with a cubic hypersurface. The line bundle $\mathcal{O}_{W}(1)$ is equal to $-2 K_{W}$ and the surface $V$ is contained in the smooth part of $W$, hence by adjunction $K_{V}=\mathcal{O}_{V}(1)$. ( $W$ is actually smooth in cases $\mathrm{A}$ and $\mathrm{B} 1$, and it is a cone over a smooth surface of $\mathbb{P}^{6}$ in case B2). It is then easy to check that $V$ has the right invariants: $K_{V}^{2}=18, p_{g}(V)=8, q(V)=0$. By construction, the threefold $W$ is the intersection of all the quadrics containing $V$.

The group $G$ acts on $W \subset \mathbb{P}^{7}$ and the action is free on $W$ outside a finite set. We choose a linearization of $\mathcal{O}_{W}(3)$ and we let $T_{1} \subset H^{0}\left(\mathcal{O}_{W}(3)\right)$ be the space of invariant sections with respect to this linearization. Then we show that the linear system $\left|T_{1}\right|$ is free on $W$, so that the general $V \in\left|T_{1}\right|$ satisfies the assumptions of Proposition 2.3 and $V / G$ is the canonical model of a Campedelli surface with $\pi_{1}^{\text {alg }}=G$. In all the examples, the map $H^{0}\left(\mathcal{O}_{\mathbb{P}^{7}}(3)\right) \rightarrow H^{0}\left(\mathcal{O}_{W}(3)\right)$ is surjective, so that $V$ is cut out on $W$ by a cubic hypersurface of $\mathbb{P}^{7}$.

Remark 3.2. In the above construction, the chosen linearization of $\mathcal{O}_{W}(3)$ induces a decomposition into $G$-eigenspaces:

$$
H^{0}\left(\mathcal{O}_{W}(3)\right)=\bigoplus_{\chi \in G^{*}} T_{\chi}
$$

For every $\chi \in G^{*}$ the linear system $\left|T_{\chi}\right|$ consists of $G$-invariant surfaces, hence one could hope to obtain surfaces with the required properties also for some character $\chi \neq 1$. It turns out (cf. Lemma 5.7) that this is not possible because for every $\chi \neq 1$ the system $\left|T_{\chi}\right|$ has base points with nontrivial stabilizer in $G$, and therefore $G$ does not act freely on the surfaces of $\left|T_{\chi}\right|$ for $\chi \neq 1$.

Remark 3.3. In each example we compute the dimension of the invariant subspace $T_{1}$ by writing down a basis of it. Alternatively, the dimension of $T_{1}$ can be computed by using the equivariant Riemann-Roch theorem ([Re1, Corollary in (8.6)]).

\subsection{Surfaces of type A}

In this example $G \cong \mathbb{Z}_{9}$. We take $W:=\mathbb{P}^{1} \times \mathbb{P}^{1} \times \mathbb{P}^{1}$ and consider homogeneous coordinates $\left(x_{0}, x_{1}\right),\left(y_{0}, y_{1}\right),\left(z_{0}, z_{1}\right)$ on $W$ and the corresponding affine coordinates $x:=x_{1} / x_{0}, y:=y_{1} / y_{0}$ and $z:=z_{1} / z_{0}$.

We fix a primitive third root $\omega$ of 1 and we let a generator $g$ of $G$ act by

$$
g:(x, y, z) \mapsto(y, z, \omega x) .
$$


Note that the fixed points of $g^{3}$ are the eight points with the three affine coordinates $x, y, z$ equal either to 0 or to $\infty$.

Set $H:=\mathcal{O}_{W}(1,1,1)$. The linear system $|H|$ embeds $W$ in $\mathbb{P}^{7}$ as a smooth threefold of degree 6. The action of $G$ extends to $\mathbb{P}^{7}$ and the projective representation of $G$ on $\mathbb{P}^{7}$ induces a linear representation on $H^{0}\left(\mathcal{O}_{\mathbb{P}^{7}}(3)\right)$, hence a linearization of $3 H$. Let $T_{1} \subset$ $H^{0}(W, 3 H)$ be the subspace of $G$-invariant elements. In affine coordinates a basis of $T_{1}$ is given by:

$$
\begin{gathered}
1, x^{3}+y^{3}+z^{3}, \quad x^{2} y+y^{2} z+\omega z^{2} x, \quad x^{2} z+\omega y^{2} x+\omega z^{2} y, \\
x^{3} y^{3}+y^{3} z^{3}+z^{3} x^{3}, \quad x^{3} y^{2} z+\omega y^{3} z^{2} x+z^{3} x^{2} y \\
x^{3} y z^{2}+\omega^{2} y^{3} z x^{2}+z^{3} x y^{2}, \quad x^{3} y^{3} z^{3} .
\end{gathered}
$$

Using this basis it is easy to check that the system $\left|T_{1}\right|$ is free of dimension 7 .

By Bertini's theorem, the general element of $\left|T_{1}\right|$ is a smooth surface on which $G$ acts freely. Let $V \in\left|T_{1}\right|$ be a surface with at most rational double points and not passing through the fixed points of $g^{3}$. By adjunction, the canonical divisor $K_{V}$ is the restriction of $H$ to $V$, hence $K_{V}^{2}=3 H^{3}=18$. The adjunction sequence gives: $p_{g}(V)=8, \chi(V)=9$, $q(V)=0$. Set $S:=V / G$. Then by Proposition 2.3 the surface $S$ is the canonical model of a numerical Campedelli surface with $\pi_{1}^{\mathrm{alg}}=G$.

Proposition 3.4. The family of surfaces of type A depends on six moduli.

Proof. Consider $V_{1}, V_{2} \in\left|T_{1}\right|$ such that the surfaces $S_{1}:=V_{1} / G$ and $S_{2}:=V_{2} / G$ are isomorphic. Since $V_{i}$ is the (algebraic) universal cover of $S_{i}$, the isomorphism $S_{1} \rightarrow S_{2}$ lifts to an isomorphism $V_{1} \rightarrow V_{2}$. Since $V_{1}$ and $V_{2}$ are canonically embedded in $\mathbb{P}^{7}$, the isomorphism $V_{1} \rightarrow V_{2}$ is induced by an automorphism $\gamma$ of $\mathbb{P}^{7}$. The threefold $W \subset \mathbb{P}^{7}$ is the intersection of all the quadrics containing $V_{i}, i=1,2$ (cf. [Ko, Theorem 3.1]), and therefore $\gamma$ maps $W$ to itself. Since every automorphism of $W$ extends to $\mathbb{P}^{7}$, we may regard $\gamma$ as an automorphism of $W$.

By construction, $\gamma$ belongs to the normalizer $\Gamma$ of $G$ in Aut $W$. Since the group $G$ is finite, the connected component $\Gamma_{1}$ of the identity in $\Gamma$ is actually contained in the centralizer of $G$. In addition, if $\gamma$ is an element of $\Gamma_{1}$, then by continuity $\gamma$ does not permute the three copies of $\mathbb{P}^{1}$. Then it is easy to see that $\Gamma_{1}$ consists of the maps of the form $(x, y, z) \mapsto(\lambda x, \lambda y, \lambda z)$ for $\lambda \in \mathbb{C}^{*}$. Hence we obtain a family of Campedelli surfaces with six moduli.

Remark 3.5. The expected dimension of the moduli space of a minimal surface of general type $S$ is $10 \chi(S)-2 K_{S}^{2}=\chi\left(T_{S}\right)$. In particular the expected dimension of the moduli space of Campedelli surfaces is equal to 6 .

Remark 3.6. J. H. Keum has kindly communicated to us an example, due to Persson, of a numerical Campedelli surface with $\pi_{1}^{\text {alg }} \cong \mathbb{Z}_{9}$. One can check that this example is given by the above construction. 


\subsection{Surfaces of type $B 1$}

In this example $G \cong \mathbb{Z}_{3}^{2}$. We take $W$ to be the flag variety $\left\{x_{0} y_{0}+x_{1} y_{1}+x_{2} y_{2}=0\right\} \subset$ $\mathbb{P}^{2} \times \mathbb{P}^{2^{*}}$. We denote by $H$ the restriction to $W$ of $\mathcal{O}_{\mathbb{P}^{2} \times \mathbb{P}^{2 *}}(1,1)$, so that $W$ is embedded by $|H|$ into $\mathbb{P}^{7}$ as a smooth threefold of degree 6 . Let $g_{1}, g_{2} \in G$ be generators acting on $\mathbb{P}^{2}$ by

$$
\left(x_{0}, x_{1}, x_{2}\right) \stackrel{g_{1}}{\mapsto}\left(x_{0}, \omega x_{1}, \omega^{2} x_{2}\right), \quad\left(x_{0}, x_{1}, x_{2}\right) \stackrel{g_{2}}{\mapsto}\left(x_{1}, x_{2}, x_{0}\right),
$$

where $\omega \neq 1$ is a third root of 1 . The induced $G$-action on $\mathbb{P}^{2} \times \mathbb{P}^{2 *}$ obviously preserves $W$ and it is easy to check that every nontrivial element of $G$ fixes finitely many points of $W$.

Notice that, although the action of $G$ on $\mathbb{P}^{2}$ is not induced by a linear action, the corresponding action on $\mathbb{P}^{2} \times \mathbb{P}^{2^{*}}$ is induced by a linear $G$-action on $H^{0}\left(\mathcal{O}_{\mathbb{P}^{2} \times \mathbb{P}^{2 *}}(1,1)\right)$. In particular, $G$ acts on $\mathcal{O}_{\mathbb{P}^{2} \times \mathbb{P}^{2}}(1,1)$, on $H$ and on their multiples. There is an exact sequence

$$
0 \rightarrow H^{0}\left(\mathcal{O}_{\mathbb{P}^{2} \times \mathbb{P}^{2 *}}(2,2)\right) \stackrel{i}{\rightarrow} H^{0}\left(\mathcal{O}_{\mathbb{P}^{2} \times \mathbb{P}^{2}}(3,3)\right) \rightarrow H^{0}(W, 3 H) \rightarrow 0 .
$$

The map $i$ is multiplication by the invariant section $x_{0} y_{0}+x_{1} y_{1}+x_{2} y_{2}$ and thus it is equivariant with respect to the action of $G$. In particular, the subspace $T_{1} \subset H^{0}(W, 3 H)$ on which $G$ acts trivially is the image of the subspace $R_{1} \subset H^{0}\left(\mathcal{O}_{\mathbb{P}^{2} \times \mathbb{P}^{2}}(3,3)\right)$ on which $G$ acts trivially. A basis of $R_{1}$, which has dimension 12 , is the following:

$$
\begin{gathered}
\left(x_{0}^{3}+x_{1}^{3}+x_{2}^{3}\right)\left(y_{0}^{3}+y_{1}^{3}+y_{2}^{3}\right), \quad\left(x_{0}^{3}+x_{1}^{3}+x_{2}^{3}\right)\left(y_{0} y_{1} y_{2}\right), \\
\left(x_{0} x_{1} x_{2}\right)\left(y_{0}^{3}+y_{1}^{3}+y_{2}^{3}\right), \quad\left(x_{0} x_{1} x_{2}\right)\left(y_{0} y_{1} y_{2}\right), \\
\left(x_{0}^{2} x_{1}+x_{1}^{2} x_{2}+x_{2}^{2} x_{0}\right)\left(y_{0}^{2} y_{1}+y_{1}^{2} y_{2}+y_{2}^{2} y_{0}\right), \\
\left(x_{1}^{2} x_{0}+x_{2}^{2} x_{1}+x_{0}^{2} x_{2}\right)\left(y_{1}^{2} y_{0}+y_{2}^{2} y_{1}+y_{0}^{2} y_{2}\right), \\
\left(x_{0}^{2} x_{1}+\omega x_{1}^{2} x_{2}+\omega^{2} x_{2}^{2} x_{0}\right)\left(y_{0}^{2} y_{1}+\omega^{2} y_{1}^{2} y_{2}+\omega y_{2}^{2} y_{0}\right), \\
\left(x_{1}^{2} x_{0}+\omega x_{2}^{2} x_{1}+\omega^{2} x_{0}^{2} x_{2}\right)\left(y_{1}^{2} y_{0}+\omega^{2} y_{2}^{2} y_{1}+\omega y_{0}^{2} y_{2}\right), \\
\left(x_{0}^{2} x_{1}+\omega^{2} x_{1}^{2} x_{2}+\omega x_{2}^{2} x_{0}\right)\left(y_{0}^{2} y_{1}+\omega y_{1}^{2} y_{2}+\omega^{2} y_{2}^{2} y_{0}\right), \\
\left(x_{1}^{2} x_{0}+\omega^{2} x_{2}^{2} x_{1}+\omega x_{0}^{2} x_{2}\right)\left(y_{1}^{2} y_{0}+\omega y_{2}^{2} y_{1}+\omega^{2} y_{0}^{2} y_{2}\right), \\
\left(x_{0}^{3}+\omega x_{1}^{3}+\omega^{2} x_{2}^{3}\right)\left(y_{0}^{3}+\omega^{2} y_{1}^{3}+\omega y_{2}^{3}\right), \quad\left(x_{0}^{3}+\omega^{2} x_{1}^{3}+\omega x_{2}^{3}\right)\left(y_{0}^{3}+\omega y_{1}^{3}+\omega^{2} y_{2}^{3}\right) .
\end{gathered}
$$

Next we write down a basis of the subspace of $H^{0}\left(\mathcal{O}_{\mathbb{P}^{2} \times \mathbb{P}^{2 *}}(2,2)\right)$ on which $G$ acts trivially:

$$
\begin{gathered}
x_{0}^{2} y_{0}^{2}+x_{1}^{2} y_{1}^{2}+x_{2}^{2} y_{2}^{2}, \\
x_{0}^{2} y_{1} y_{2}+x_{1}^{2} y_{0} y_{2}+x_{2}^{2} y_{0} y_{1}, \quad x_{1} x_{2} y_{0}^{2}+x_{0} x_{2} y_{1}^{2}+x_{0} x_{1} y_{2}^{2}, \\
x_{0} x_{1} y_{0} y_{1}+x_{1} x_{2} y_{1} y_{2}+x_{2} x_{0} y_{2} y_{0} .
\end{gathered}
$$

It follows that the space $T_{1}$ has dimension $12-4=8$. Let $z_{0}, \ldots, z_{8}$ be a basis of $H^{0}\left(\mathcal{O}_{\mathbb{P}^{2} \times \mathbb{P}^{2 *}}(1,1)\right)$ on which $G$ acts diagonally. Then $z_{0}^{3}, \ldots, z_{8}^{3}$ restrict on $W$ to elements of $T_{1}$, hence the linear system $\left|T_{1}\right|$ is free. 
By Bertini's theorem, the general $V \in\left|T_{1}\right|$ is a smooth surface on which $G$ acts freely. Let $V \in\left|T_{1}\right|$ be a surface with at most rational double points and not passing through any point fixed by a nontrivial element of $G$. By adjunction, the canonical divisor $K_{V}$ is the restriction of $H$ to $V$, hence $K_{V}^{2}=3 H^{3}=18$. The adjunction sequence for $V \subset W$ gives: $p_{g}(V)=8, \chi(V)=9, q(V)=0$. Set $S:=V / G$. Then by Proposition 2.3 the surface $S$ is the canonical model of a numerical Campedelli surface with $\pi_{1}^{\text {alg }}=G$.

To compute the number of moduli for surfaces of type B1 we need the following description of Aut $W$ :

Lemma 3.7. There is an exact sequence

$$
0 \rightarrow \text { Aut } \mathbb{P}^{2} \stackrel{i}{\rightarrow} \text { Aut } W \stackrel{\sigma}{\rightarrow} \mathbb{Z}_{2} \rightarrow 0 .
$$

In particular $G$, being of order 9 , can be identified with a subgroup of Aut $\mathbb{P}^{2}$.

Proof. Every automorphism $g$ of $\mathbb{P}^{2}$ extends naturally to an automorphism of $\mathbb{P}^{2} \times \mathbb{P}^{2^{*}}$ that preserves the flag variety $W$. The Picard group of $W$ is isomorphic to $\mathbb{Z}^{2}$, since $W$ is a $\mathbb{P}^{1}$-bundle over $\mathbb{P}^{2}$. Set $H_{1}:=\mathcal{O}_{W}(1,0), H_{2}:=\mathcal{O}_{W}(0,1)$. The classes of $H_{1}$ and $H_{2}$ are a basis of $H^{2}(W, \mathbb{Q})$ and they satisfy $H_{1}^{3}=H_{2}^{3}=0, H_{1} K_{W}^{2}=H_{2} K_{W}^{2}=12$. It is easy to check that these numerical conditions characterize $H_{1}$ and $H_{2}$. Hence every automorphism $\gamma$ of $W$ either preserves the classes of $H_{1}$ and $H_{2}$, or exchanges them. The map $\sigma:$ Aut $W \rightarrow \mathbb{Z}_{2}$ sends $\gamma$ to 0 in the former case and to 1 in the latter. Clearly the map $\sigma$ is not trivial and its kernel contains Aut $\mathbb{P}^{2}$.

Now let $g \in \operatorname{ker} \sigma$. Then $g$ induces an automorphism $g_{1}$ of $\mathbb{P}^{2}=\left|H_{1}\right|^{*}$ and an automorphism $g_{2}$ of $\mathbb{P}^{2 *}=\left|H_{2}\right|^{*}$ such that the automorphism $g_{1} \times g_{2}$ of $\mathbb{P}^{2} \times \mathbb{P}^{2^{*}}$ restricts to $g$ on $W$. Since the flag variety $W$ is preserved, it follows that $g_{2}$ is equal to ${ }^{t} g_{1}^{-1}$, hence $g$ is in the subgroup Aut $\mathbb{P}^{2}$.

Proposition 3.8. The family of surfaces of type B1 depends on seven moduli.

Proof. By the argument in the proof of Proposition 3.4, it is enough to show that the centralizer $\Gamma$ of $G$ in Aut $W$ is discrete.

Let $\Gamma_{1}$ be the connected component of the identity in $\Gamma$ and let $\gamma \in \Gamma_{1}$. By Lemma 3.7 and by continuity, $\gamma$ is induced by an element of Aut $\mathbb{P}^{2}$, which we again denote by $\gamma$. Since $\gamma g \gamma^{-1}=g$ for every $g \in G, \gamma$ permutes the fixed points of $g$ and hence, again by continuity, the fixed points of $g$ on $\mathbb{P}^{2}$ are also fixed points of $\gamma$. Since the set of points in $\mathbb{P}^{2}$ that are fixed by a nontrivial $g \in G$ consists of twelve points in general position, $\gamma$ is the identity.

Remark 3.9. As we recalled in Remark 3.5. the expected dimension of the moduli space of Campedelli surfaces is 6 . To the best of our knowledge, the surfaces of type B1 are the only known example where the actual dimension exceeds the expected one.

Remark 3.10. The first known example of a numerical Campedelli surface with torsion $\mathbb{Z}_{3}^{2}$ is due to Xiao Gang [Xi1, Example 4.11]. A particular case of the construction above has been kindly communicated to us by JongHae Keum, who attributes it to Xiao Gang and Beauville. See also [CMP, Example 2 of $§ 5]$. 


\subsection{Surfaces of type $B 2$}

This is the second example with $G \cong \mathbb{Z}_{3}^{2}$. It is also the only example in which $W$ is singular.

Let $\varepsilon: \Sigma \rightarrow \mathbb{P}^{2}$ be the blowup at the three coordinate points. The $G$-action on $\mathbb{P}^{2}$ defined in (3.3) extends to a $G$-action on $\Sigma$.

The action on $\mathbb{P}^{2}$ does not lift to a linear action on $H^{0}\left(\mathcal{O}_{\mathbb{P}^{2}}(1)\right)$, but it induces a natural linear action of $G$ on $H^{0}\left(\mathcal{O}_{\mathbb{P}^{2}}(3)\right)$ that preserves the subspace $T$ of sections vanishing at the coordinate points. Let $\chi_{1} \in G^{*}$ be the character such that $\chi_{1}\left(g_{1}\right)=\omega, \chi_{1}\left(g_{2}\right)=1$, and let $\chi_{2}$ be the character such that $\chi_{2}\left(g_{1}\right)=1, \chi_{2}\left(g_{2}\right)=\omega$. The following is a basis of the vector space $T$ of eigenvectors for the $G$-action. We write the corresponding character next to each element:

$$
\begin{array}{rll}
x_{0}^{2} x_{1}+x_{1}^{2} x_{2}+x_{2}^{2} x_{0} & (1,0) ; & x_{1}^{2} x_{0}+x_{2}^{2} x_{1}+x_{0}^{2} x_{2} \\
x_{0}^{2} x_{1}+\omega x_{1}^{2} x_{2}+\omega^{2} x_{2}^{2} x_{0} & (1,2) ; & x_{1}^{2} x_{0}+\omega x_{2}^{2} x_{1}+\omega^{2} x_{0}^{2} x_{2} \\
x_{0}^{2} x_{1}+\omega^{2} x_{1}^{2} x_{2}+\omega x_{2}^{2} x_{0} & (1,1) ; & x_{1}^{2} x_{0}+\omega^{2} x_{2}^{2} x_{1}+\omega x_{0}^{2} x_{2} \\
x_{0} x_{1} x_{2} & (0,0) . &
\end{array}
$$

We fix homogeneous coordinates $\left(z_{i j}\right)$ on $\mathbb{P}^{7}$, for $(i, j) \in \mathbb{Z}_{3}^{2} \backslash\{(0,0)\}$, and we let $G$ act on $z_{i j}$ via the character $\chi_{1}^{i} \chi_{2}^{j}$. Let $P$ be the point whose only nonzero coordinate is $z_{01}$ and identify $\mathbb{P}^{6}$ with the $G$-invariant hyperplane $z_{01}=0$. The rational map $\mathbb{P}^{2} \rightarrow \mathbb{P}^{6} \subset \mathbb{P}^{7}$ defined by

$$
\begin{array}{ll}
z_{12}=x_{0}^{2} x_{1}+x_{1}^{2} x_{2}+x_{2}^{2} x_{0}, & z_{22}=x_{1}^{2} x_{0}+x_{2}^{2} x_{1}+x_{0}^{2} x_{2}, \\
z_{11}=x_{0}^{2} x_{1}+\omega x_{1}^{2} x_{2}+\omega^{2} x_{2}^{2} x_{0}, & z_{21}=x_{1}^{2} x_{0}+\omega x_{2}^{2} x_{1}+\omega^{2} x_{0}^{2} x_{2}, \\
z_{10}=x_{0}^{2} x_{1}+\omega^{2} x_{1}^{2} x_{2}+\omega x_{2}^{2} x_{0}, & z_{20}=x_{1}^{2} x_{0}+\omega^{2} x_{2}^{2} x_{1}+\omega x_{0}^{2} x_{2}, \\
z_{02}=x_{0} x_{1} x_{2} &
\end{array}
$$

induces a $G$-equivariant embedding $\Sigma \rightarrow \mathbb{P}^{6} \subset \mathbb{P}^{7}$. Notice that the embedding is not induced by a $G$-equivariant embedding $T \rightarrow H^{0}\left(\mathcal{O}_{\mathbb{P}^{7}}(1)\right)$. Indeed, by 3.4 the representation of $G$ on $T$ differs from the one induced by the representation on $H^{0}\left(\mathcal{O}_{\mathbb{P}^{7}}(1)\right)$ by multiplication by $\chi_{2}^{2}$. This is due to the fact that we have chosen the linearization of $H^{0}\left(\mathcal{O}_{\mathbb{P}^{7}}(1)\right)$ so as to agree with the one induced by the identification with $H^{0}\left(V, K_{V}\right)$, where $V$ is any of the surfaces that we are constructing. Since by Proposition 2.4 the space $H^{0}\left(V, K_{V}\right)$ has no trivial subrepresentation, there is no $G$-equivariant embedding $T \hookrightarrow H^{0}\left(\mathcal{O}_{\mathbb{P}^{7}}(1)\right)$.

Let $W$ be the cone over $\Sigma$ with vertex $P$. Then $W$ is a singular threefold of degree 6 and it is mapped to itself by the $G$-action. We now show that every nontrivial element of $G$ has only isolated fixed points on $W$.

Notice that every fixed point of $g \in G$ on $W \backslash\{P\}$ lies on the line joining the vertex $P$ to a fixed point of $g$ on $\Sigma$.

Consider first an element $g \notin\left\langle g_{1}\right\rangle$. One checks that $g$ has three isolated fixed points $Q_{1}, Q_{2}, Q_{3}$ on $\Sigma$, none of which satisfies $z_{02}=0$. By assumption, $\chi_{2}(g)=\omega^{a}$ with 
$a=1$ or $a=2$. Hence $g$ acts by multiplying the coordinate $z_{01}$ by $\omega^{a}$ and the coordinate $z_{02}$ by $\omega^{2 a}$. Thus $g$ acts nontrivially on the line $P Q_{i}$ for $i=1,2,3$, and $P, Q_{1}, Q_{2}, Q_{3}$ are the only fixed points of $g$ on $W$. Consider now $g=g_{1}$. The fixed points of $g_{1}$ on $\Sigma$ are the six points that lie on two of the -1 -curves contained in $\Sigma$. If $e_{0}$ is the strict transform of the line $x_{0}=0$ (say), then $e_{0}$ is embedded in $\mathbb{P}^{6}$ by the following map:

$$
\begin{gathered}
z_{02}=0, \quad z_{12}=x_{1}, \quad z_{22}=x_{2}, \quad z_{10}=\omega^{2} x_{1}, \\
z_{20}=\omega^{2} x_{2}, \quad z_{11}=\omega x_{1}, \quad z_{21}=\omega x_{2} .
\end{gathered}
$$

The two fixed points of $g_{1}$ on $e_{0}$ correspond to the points $Q_{1}$ of coordinates $x_{1}=1$, $x_{2}=0$ and $Q_{2}$ of coordinates $x_{1}=0, x_{2}=1$. Hence the action of $g_{1}$ on the lines $P Q_{i}$ is nontrivial (for $i=1,2$ ). Since the set of fixed points of $g_{1}$ on $\Sigma$ is the union of the $G$-orbits of $Q_{1}$ and $Q_{2}$, the previous computation shows that the fixed points of $g_{1}$ on $W$ are $P$ and the points $Q_{1}, \ldots, Q_{6} \in \Sigma$.

Consider now the subspace $T_{1} \subset H^{0}\left(\mathcal{O}_{W}(3)\right)$ on which the $G$-action is trivial. If we set $z:=z_{01}$, then we can write

$$
H^{0}\left(\mathcal{O}_{W}(3)\right)=\left\langle z^{3}\right\rangle \oplus z^{2} H^{0}\left(\mathcal{O}_{\Sigma}(1)\right) \oplus z H^{0}\left(\mathcal{O}_{\Sigma}(2)\right) \oplus H^{0}\left(\mathcal{O}_{\Sigma}(3)\right),
$$

and this decomposition is compatible with the $G$-action. We identify $H^{0}\left(\mathcal{O}_{\Sigma}(i)\right)$ with the space of homogeneous polynomials of degree $3 i$ in $x_{0}, x_{1}, x_{2}$ that vanish of order at least $i$ at the coordinate points of $\mathbb{P}^{2}$. Then the following is a basis of $T_{1}$ :

$$
\begin{gathered}
z^{3}, \quad z\left(x_{0}^{4} x_{1} x_{2}+\omega^{2} x_{1}^{4} x_{2} x_{0}+\omega x_{2}^{4} x_{0} x_{1}\right), \quad z\left(x_{0}^{3} x_{1}^{3}+\omega^{2} x_{1}^{3} x_{2}^{3}+\omega x_{2}^{3} x_{0}^{3}\right), \\
x_{0}^{6} x_{1}^{3}+x_{1}^{6} x_{2}^{3}+x_{2}^{6} x_{0}^{3}, \quad x_{0}^{6} x_{2}^{3}+x_{1}^{6} x_{0}^{3}+x_{2}^{6} x_{1}^{3}, \quad x_{0}^{5} x_{1}^{2} x_{2}^{2}+x_{1}^{5} x_{2}^{2} x_{0}^{2}+x_{2}^{5} x_{0}^{2} x_{1}^{2}, \\
x_{0}^{4} x_{1}^{4} x_{2}+x_{1}^{4} x_{2}^{4} x_{0}+x_{2}^{4} x_{0}^{4} x_{1}, \quad x_{0}^{3} x_{1}^{3} x_{2}^{3} .
\end{gathered}
$$

The dimension of $T_{1}$ is equal to 8 and the corresponding linear system $\left|T_{1}\right|$ is free, since all the cubic powers of the homogeneous coordinates $z_{i j}$ of $\mathbb{P}^{7}$ restrict on $W$ to elements of $T_{1}$.

Let $V \in\left|T_{1}\right|$ be a surface with at most rational double points and not passing through the points fixed by a nontrivial element of $G$. Set $W_{0}:=\operatorname{Proj}\left(\mathcal{O}_{\Sigma} \oplus \mathcal{O}_{\Sigma}(1)\right)$ and let $H$ be the tautological bundle. The system $|H|$ gives a morphism $W_{0} \rightarrow W$ that contracts the divisor $E$ "at infinity" to the vertex $P$ of the cone and restricts to an isomorphism $W_{0} \backslash E \rightarrow W \backslash\{P\}$. The surface $V$ does not contain $P$, hence it can be identified with an element of $|3 H|$ on $W_{0}$, which we again denote by $V$. Using adjunction on $W_{0}$, one sees that the canonical divisor $K_{V}$ is the restriction of $H$ to $V$, hence $K_{V}^{2}=3 H^{3}=18$. The adjunction sequence gives: $p_{g}(V)=8, \chi(V)=9, q(V)=0$. Set $S:=V / G$. Then by Proposition 2.3. $S$ is the canonical model of a numerical Campedelli surface with $\pi_{1}^{\text {alg }}=G$.

Proposition 3.11. The family of surfaces of type B2 depends on six moduli. 
Proof. By the argument in the proof of Proposition 3.4, it is enough to show that the centralizer $\Gamma$ of $G$ in Aut $W$ is discrete.

Let $\Gamma_{1}$ be the connected component of the identity in $\Gamma$ and let $\gamma \in \Gamma_{1}$. The $\mathbb{P}^{6}$ containing $\Sigma$ is mapped to itself by $\gamma$, and by continuity $\gamma$ maps each of the six lines of $\Sigma$ to itself. Hence the restriction of $\gamma$ to $\Sigma$ is induced by an element of Aut $\mathbb{P}^{2}$, which we denote by $\gamma_{0}$. Arguing as in the proof of Proposition 3.8 one shows that $\gamma_{0}$ is the identity on $\mathbb{P}^{2}$, hence $\gamma$ restricts to the identity on $\mathbb{P}^{6} \supset \Sigma$. Thus the connected component of the identity in $\Gamma$ consists of the automorphisms that multiply $z_{01}$ by $\lambda \in \mathbb{C}^{*}$ and do not change the remaining homogeneous coordinates. Hence the surfaces of type B2 depend on $7-1=6$ moduli.

\subsection{A common construction}

We describe a common construction of the surfaces of type B1 and B2, suggested to us by Miles Reid.

Denote by $Z \subset \mathbb{P}^{8}$ the Segre embedding of $\mathbb{P}^{2} \times \mathbb{P}^{2^{*}}$ and let $G$ act on $Z \subset \mathbb{P}^{8}$ as in the case of surfaces of type B1. It is easy to check that the corresponding representation on $H^{0}\left(\mathbb{P}^{8}, \mathcal{O}_{\mathbb{P} 8}(1)\right)$ is the sum of the nine characters of $G$. We identify the group of characters $G^{*}$ with $\mathbb{Z}_{3}^{2}$ as in $\$ 3.3$ and we denote by $z_{i j}$ homogeneous coordinates on $\mathbb{P}^{8}$ such that $G$ acts on $z_{i j}$ via the character $(i, j)$. The hyperplane $z_{00}=0$ cuts out on $Z$ the flag variety $x_{0} y_{0}+x_{1} y_{1}+x_{2} y_{2}=0$, and the hyperplane $z_{01}=0$ cuts out on $Z$ the invariant hypersurface $x_{0} y_{0}+\omega^{2} x_{1} y_{1}+\omega^{2} x_{2} y_{2}=0$. Set $\Sigma:=Z \cap\left\{z_{00}=z_{01}=0\right\}$. The surface $\Sigma$ is a smooth $G$-invariant Del Pezzo surface of degree 6 in $\mathbb{P}^{6}$ and the $G$-action on $\Sigma$ coincides with the action given in 3.3 .

Consider now homogeneous coordinates $z_{i j}, w$ on $\mathbb{P}^{9}$, identify $\mathbb{P}^{8}$ with the hyperplane $w=0$ and let $K$ be the cone over $Z$ with vertex $P:=[0, \ldots, 0,1]$. Extend the $G$-action to $\mathbb{P}^{9}$ by letting $G$ act on $w$ via the character $(0,1)$.

Set $R_{1}:=H^{0}\left(\mathcal{O}_{\mathbb{P}^{9}}(3)\right)^{G}$. The cubes $z_{i j}^{3}, w^{3}$ of the coordinates belong to $R_{1}$, hence the system $\left|R_{1}\right|$ is free. Let now $V$ be the surface obtained by intersecting $K$ with an element of $\left|R_{1}\right|$, the hyperplane $z_{00}=0$ and a hyperplane of the form $\lambda z_{01}+\mu w=0$. The general $V$ is smooth by Bertini's theorem. Assume that $V$ has at most canonical singularities. If $\lambda=0$, then $V / G$ is a surface of type B2 and it is clear that every surface of type B2 can be obtained in this way. If $\lambda \neq 0$, then $V / G$ is a surface of type $\mathrm{B} 1$, and every surface of type B1 can be obtained in this way, for instance taking $\mu=0$.

In particular, this construction proves the following:

Proposition 3.12. Let $S$ be a surface of type B2. Then $S$ is a limit of surfaces of type $B 1$.

Remark 3.13. In all three cases the general hyperplane section of $W$ is the smooth Del Pezzo surface $\Sigma$ of degree 6 in $\mathbb{P}^{6}$, hence $W$ can be deformed to the cone over $\Sigma$. By the above construction, in case B1 it is possible to preserve the $\mathbb{Z}_{3}^{2}$-action in the deformation. This is not possible in case A. Indeed, the limit cone would have a smooth $\mathbb{Z}_{9}$-invariant hyperplane section, while $\mathbb{P}^{1} \times \mathbb{P}^{1} \times \mathbb{P}^{1}$ has no such section. 


\section{Geometry and moduli}

In this section we study some geometrical properties of numerical Campedelli surfaces with $\pi_{1}^{\text {alg }}$ of order 9 and of their moduli space.

\subsection{Moduli}

Notice first of all the following consequence of Theorem 3.1

Proposition 4.1. The canonical divisor $K_{S}$ of a general numerical Campedelli surface $S$ with $\pi_{1}^{\mathrm{alg}}(S)$ of order 9 is ample.

Proof. The statement is immediate by Theorem 3.1 and by the description of the three families of surfaces in $\S 3$.

The next result describes the moduli space of Campedelli surfaces with $\left|\pi_{1}^{\mathrm{alg}}\right|=9$ :

Theorem 4.2. Let $\mathcal{M}$ be the moduli space of numerical Campedelli surfaces, let $\mathcal{M}_{A} \subset$ $\mathcal{M}$ be the subset of surfaces with $\pi_{1}^{\mathrm{alg}}=\mathbb{Z}_{9}$ and let $\mathcal{M}_{B} \subset \mathcal{M}$ be the subset of surfaces with $\pi_{1}^{\text {alg }}=\mathbb{Z}_{3}^{2}$. Then:

(i) $\mathcal{M}_{A}$ and $\mathcal{M}_{B}$ are connected components of $\mathcal{M}$;

(ii) $\mathcal{M}_{A}$ is irreducible of dimension 6;

(iii) $\mathcal{M}_{B}$ is irreducible of dimension 7.

Proof. Since $\pi_{1}^{\text {alg }}$ is the profinite completion of $\pi_{1}$, it is a topological invariant. Thus $\mathcal{M}_{A}$ and $\mathcal{M}_{B}$ are open and closed in $\mathcal{M}$. By Theorem 3.1 (i), the points of $\mathcal{M}_{A}$ correspond to the surfaces of type $\mathrm{A}$, hence $\mathcal{M}_{A}$ is irreducible of dimension 6 by Proposition 3.4 By Theorem 3.1 (ii), the points of $\mathcal{M}_{B}$ correspond to the surfaces of type B1 and $\mathrm{B} 2$. By Proposition 3.12, the surfaces of type B2 lie in the closure of the set of surfaces of type B1. In turn, the surfaces of type B1 form an irreducible subset of dimension 7 by Proposition 3.8

\subsection{The topological fundamental group}

Having an explicit construction of Campedelli surfaces with $\left|\pi_{1}^{\text {alg }}\right|=9$ allows one to determine also the topological fundamental group.

Proposition 4.3. Let $S$ be a numerical Campedelli surface such that $\pi_{1}^{\mathrm{alg}}(S)$ has order 9 (Tors $S$ has order 9 ). Then $\pi_{1}(S)=\pi_{1}^{\text {alg }}(S)$.

Proof. The group Tors $S$ is the largest abelian quotient of $\pi_{1}^{\text {alg }}(S)$. Thus, by Proposition 2.1. the group Tors $S$ has order 9 if and only if $\pi_{1}^{\mathrm{alg}}(S)$ has order 9 . 
Assume that this is the case. Then it is enough to prove the statement for one surface in each irreducible component of the moduli space of numerical Campedelli surfaces with $\pi_{1}^{\text {alg }}$ of order 9. If $S$ is a surface of type A or B1 with $K_{S}$ ample, then $V$ is a smooth ample divisor inside a smooth simply connected threefold. Hence $V$ is simply connected by the Lefschetz theorem on hyperplane sections and it is actually the universal cover of $S$. By Theorem 4.2 , this proves the statement.

\subsection{The bicanonical system}

We now study the bicanonical system.

Theorem 4.4. Let $S$ be a numerical Campedelli surface such that $\pi_{1}^{\mathrm{alg}}(S)$ has order 9 and let $\Gamma$ be the base locus of the bicanonical system $\left|2 K_{S}\right|$. Then:

(i) if $S$ is of type $A$, then $\Gamma$ consists of two points;

(ii) if $S$ is of type $B 1$, then $\Gamma$ is empty;

(iii) if $S$ is of type $B 2$, then $\Gamma$ consists of two points.

Remark 4.5. Recall that, excluding the case $K^{2}=1, p_{g}=0$, the bicanonical map of a minimal surface of general type is generically finite ([Xi2] $)$, and it is a morphism if either $p_{g}>0$ or $K^{2}>4$ (cf. [Ci]). To our knowledge, surfaces of type A and of type B2 are the only known examples of surfaces of general type with $K^{2}>1$ whose bicanonical map is not a morphism.

Proof of Theorem 4.4 We use the notation introduced in the previous sections.

Denote by $X$ the canonical model of $S$, by $p: V \rightarrow X$ the quotient map and by $\varepsilon: S \rightarrow X$ the minimal resolution. Since $\left|2 K_{S}\right|=\varepsilon^{*}\left|2 K_{X}\right|$, we study the base locus of $\left|2 K_{X}\right|$. Assume that $S$ is a surface of type A. In this case $W$ is the Segre embedding of $\mathbb{P}^{1} \times \mathbb{P}^{1} \times \mathbb{P}^{1}$ in $\mathbb{P}^{7}$ and a generator $g \in \mathbb{Z}_{9}$ acts on $W$ as $(3.1)$. The $G$-action on $W$ induces an action on $H^{0}\left(\mathcal{O}_{W}(1)\right)$ which is determined only up to multiplication by an element of $G^{*}$. Let $\zeta$ be a primitive 9-th root of 1 and denote by $\chi$ the character such that $\chi(g)=\zeta$. If we require that the representation on $H^{0}\left(\mathcal{O}_{W}(1)\right)$ does not contain the trivial character, then the representation is determined and the following is a basis such that $G$ uniquely acts on $z_{j}$ as multiplication by $\chi^{j}$ :

$$
\begin{array}{llrl}
z_{4} & :=z+\zeta y+\zeta^{2} x, & z_{7}:=z+\zeta^{4} y+\zeta^{8} x, \\
z_{1}:=z+\zeta^{7} y+\zeta^{5} x, & z_{5}:=x y+\zeta^{7} y z+\zeta^{8} x z, \\
z_{8}:=x y+\zeta^{4} y z+\zeta^{2} x z, & z_{2}:=x y+\zeta y z+\zeta^{5} x z, \\
z_{3}:=1, & z_{6}:=x y z .
\end{array}
$$

By Proposition 2.4 this choice of action on $H^{0}\left(\mathcal{O}_{W}(1)\right)$ gives a $G$-isomorphism of $H^{0}\left(\mathcal{O}_{W}(1)\right)$ with $H^{0}\left(K_{V}\right)=H^{0}\left(K_{Y}\right)$.

The threefold $W$ is projectively normal, hence $V$, being cut out on $W$ by a cubic hypersurface, is also projectively normal. Thus $p^{*} H^{0}\left(2 K_{X}\right)$ is generated by the restrictions 
to $V$ of the four quadrics $z_{i} z_{9-i}$ for $i=1, \ldots, 4$. It is not difficult to check that the zero locus on $W$ of these quadrics is the union of the following curves:

$$
\begin{array}{lll}
L_{1}:=\{x=y=0\}, & L_{2}:=\{x=z=0\}, & L_{3}:=\{y=z=0\}, \\
L_{4}:=\{x=y=\infty\}, & L_{5}:=\{x=z=\infty\}, & L_{6}:=\{y=z=\infty\} .
\end{array}
$$

The surface $V$ does not contain any of the curves $L_{i}$, since the $G$-action on $V$ is free, while the points $(0,0,0)=L_{1} \cap L_{2} \cap L_{3}$ and $(\infty, \infty, \infty)=L_{4} \cap L_{5} \cap L_{6}$ are fixed points of $G$. Moreover, each of the $L_{i}$ is mapped to itself by $g^{3}$. It follows that for every $i=1, \ldots, 6$ the set $V \cap L_{i}$ consists of three distinct points, which form an orbit for the action of $g^{3}$. In particular, for $i=1, \ldots, 6$ the points of $V \cap L_{i}$ are smooth for $V$. Hence $p^{*}\left|2 K_{X}\right|$ has 18 base points and $\left|2 K_{X}\right|$ has two base points. Since the base points of $\left|2 K_{X}\right|$ are smooth points of $X$, also $\left|2 K_{S}\right|$ has two base points.

Assume now that $S$ is a surface of type B1 or B2, so that $G=\mathbb{Z}_{3}^{2}$. In both cases the $G$ action on $W$ and on $\mathbb{P}^{7}$ is induced by the $G$-action on $\mathbb{P}^{2}$ of $(3.3)$. Let $\chi_{1}$ be the character of $G$ such that $\chi_{1}\left(g_{1}\right)=\omega, \chi_{1}\left(g_{2}\right)=1$, and let $\chi_{2}$ be the character such that $\chi_{2}\left(g_{1}\right)=1$, $\chi_{2}\left(g_{2}\right)=\omega$. Denote by $\left(z_{i j}\right)$, for $(i, j) \in \mathbb{Z}_{3}^{2} \backslash\{0\}$, homogeneous coordinates on $\mathbb{P}^{7}$ such that $G$ acts on $z_{i j}$ as multiplication by $\chi_{1}^{i} \chi_{2}^{j}$. Arguing as in the case of surfaces of type A, we find that $p^{*}\left|2 K_{X}\right|$ is generated by the restrictions to $V$ of the following quadrics:

$$
z_{10} z_{20}, z_{01} z_{02}, z_{11} z_{22}, z_{12} z_{21} \text {. }
$$

Assume that $S$ is of type B1. Then up to multiplication by nonzero scalars we have the following equalities on $W$ :

$$
\begin{array}{ll}
z_{20}:=x_{0} y_{0}+\omega x_{1} y_{1}+\omega^{2} x_{2} y_{2} ; & z_{10}:=x_{0} y_{0}+\omega^{2} x_{1} y_{1}+\omega x_{2} y_{2} \\
z_{02}:=x_{0} y_{1}+x_{1} y_{2}+x_{2} y_{0} ; & z_{01}:=x_{0} y_{2}+x_{1} y_{0}+x_{2} y_{1} \\
z_{22}:=x_{0} y_{1}+\omega x_{1} y_{2}+\omega^{2} x_{2} y_{0} ; & z_{21}:=x_{0} y_{2}+\omega x_{1} y_{0}+\omega^{2} x_{2} y_{1} \\
z_{12}:=x_{0} y_{1}+\omega^{2} x_{1} y_{2}+\omega x_{2} y_{0} ; & z_{11}:=x_{0} y_{2}+\omega^{2} x_{1} y_{0}+\omega x_{2} y_{1} .
\end{array}
$$

An easy computation shows that the zero locus $\Sigma_{i j}$ of $z_{i j}$ on $W$ is a smooth surface for every $(0,0) \neq(i, j) \in \mathbb{Z}_{3}^{2}$. Hence $\Sigma_{i j}$ is a smooth Del Pezzo surface of degree 6 and, in particular, it contains six lines, which we denote by $e_{i}$ for $i \in \mathbb{Z}_{6}$. The lines $e_{i}$ form a "hexagon", namely we can arrange the indices in such a way that $e_{i} \cap e_{j}$ is a point if $i-j= \pm 1$ and it is empty otherwise. The points $R_{i}:=e_{i} \cap e_{i+1}$ are distinct for $i \in \mathbb{Z}_{6}$. The group $G$ preserves the set $\left\{e_{1}, \ldots, e_{6}\right\}$. Considering the intersection numbers $e_{i} e_{j}$, one sees that an element of $G$ either maps each $e_{i}$ to itself, or induces a "rotation" of order 3 of the hexagon. Notice that the whole group $G$ cannot act trivially on $\left\{e_{1}, \ldots, e_{6}\right\}$, since the points $R_{1}, \ldots, R_{6}$ would then be fixed by all the group.

Hence, if we denote by $\varepsilon_{i j}: \Sigma_{i j} \rightarrow \mathbb{P}^{2}$ the birational morphism that blows down $e_{1}, e_{3}, e_{5}$, then the $G$-action on $\Sigma_{i j}$ descends to a $G$-action on $\mathbb{P}^{2}$. Since $G$ acts freely on $W$, and hence on $\Sigma_{i j}$, outside a finite set, by Lemma 5.5 below it follows that the action of $G$ on $\Sigma_{i j}$ is the one described in $\$ 3.3$. In particular, the $G$-invariant hyperplane sections of $\Sigma_{i j}$ are those defined by the equations 3.5. In particular all these sections 
are irreducible except one, which is the union of the six lines of $\Sigma_{i j}$. (It is not difficult to convince oneself that the reducible section is given by $z_{2 i} 2 j=0$ ).) So for every choice of $\left(i_{1}, j_{1}\right), \ldots,\left(i_{4}, j_{4}\right) \in \mathbb{Z}_{3}^{2}$ such that $\left(i_{r}, j_{r}\right)+\left(i_{s}, j_{s}\right) \neq(0,0)$ for $1 \leq r, s \leq 4$, the intersection of $W$ with the subspace $z_{i_{1} j_{1}}=\cdots=z_{i_{4} j_{4}}=0$ is a $G$-invariant set, properly contained in a hyperplane section of the smooth elliptic curve $\Sigma_{i_{1} j_{1}} \cap \Sigma_{i_{2} j_{2}}$. Hence this set has at most six points, each of which has nontrivial stabilizer. So the base locus $\Gamma_{0}$ on $W$ of the quadrics 4.2 ) is a finite set (it is easy to check that $\Gamma_{0}$ is nonempty) and every point of $\Gamma_{0}$ has nontrivial stabilizer. Since $G$ acts freely on $V$, it follows that $V \cap \Gamma_{0}=\emptyset$. So the system $p^{*}\left|2 K_{X}\right|$ is free and, as a consequence, $\left|2 K_{X}\right|$ and $\left|2 K_{S}\right|$ are also free.

Assume now that $S$ is of type B2. We use the notation of $\$ 3.3$ and, as in the case of surfaces of type B1, we study the zero set on $W$ of the quadrics (4.2). Write $\Sigma$ for the intersection of $W$ with $z_{01}=0$, so $\Sigma$ is the only $G$-invariant section of $W$ not containing the vertex $P$ of $W$. Take $\left(i_{1}, j_{1}\right), \ldots,\left(i_{4}, j_{4}\right) \in \mathbb{Z}_{3}^{2}$ such that $\left(i_{r}, j_{r}\right)+\left(i_{s}, j_{s}\right) \neq(0,0)$ for $1 \leq r, s \leq 4$. Up to permuting the indices, we may assume that $\left(i_{1}, j_{1}\right)=(0,1)$ or $\left(i_{1}, j_{1}\right)=(0,2)$. If $\left(i_{1}, j_{1}\right)=(0,1)$ then we may argue as in the previous case and show that the intersection of $W$ with the subspace $z_{01}=z_{i_{2} j_{2}}=z_{i_{3} j_{3}}=z_{i_{4} j_{4}}=0$ is a finite set each of whose points has nontrivial stabilizer.

Now assume that $\left(i_{1}, j_{1}\right)=(0,2)$. Then the intersection of $W$ with the subspace $z_{02}=z_{i_{2} j_{2}}=z_{i_{3} j_{3}}=z_{i_{4} j_{4}}=0$ is the join of $P$ and of the intersection of $\Sigma$ with the subspace $z_{02}=z_{i_{2} j_{2}}=z_{i_{3} j_{3}}=z_{i_{4} j_{4}}=0$. By the formulae (3.5), as the indices $\left(i_{2}, j_{2}\right),\left(i_{3}, j_{3}\right),\left(i_{4}, j_{4}\right)$ vary one obtains the six points of intersection of the six lines of $\Sigma$. Summing up, the zero set on $W$ of the quadrics $(4.2)$ is the union of finitely many points with nontrivial stabilizer and of six rulings of $W$. The same argument that we have used for surfaces of type A shows that $\left|2 K_{X}\right|$ has two base points, which are smooth for $X$, and thus $\left|2 K_{S}\right|$ also has two base points.

\section{Proof of the classification}

This section proves the classification theorem 3.1 We use freely the notation and the assumptions of $\S 2$.

Recall that the universal cover $Y$ of $S$ satisfies $K_{Y}^{2}=3 p_{g}(Y)-6$. A detailed study of surfaces satisfying this relation has been carried out by Konno ([Ko] $)$. We recall here some of his results. By Proposition 2.2 (ii), the surface $Y$ belongs to type I in Konno's classification. Denote by $V \subset \mathbb{P}^{7}$ the image of the canonical map $\varphi: Y \rightarrow \mathbb{P}^{7}$ and by $W$ the intersection of all the quadrics of $\mathbb{P}^{7}$ containing $V$. The natural linear $G$-action on $H^{0}\left(Y, K_{Y}\right)$ descends to a $G$-action on $\mathbb{P}^{7}=\mathbb{P}\left(H^{0}\left(Y, K_{Y}\right)^{*}\right)$ that preserves $V$ and $W$.

Following Fujita, we define the $\Delta$-genus of a projective variety $W$ of $\mathbb{P}^{n}$ as $\operatorname{deg} W+$ $\operatorname{dim} W-n-1$.

Proposition 5.1 ([|Ko]$)$. The variety $W$ has dimension 3 and $\Delta$-genus 0 or 1 . Furthermore:

(i) if $W$ has $\Delta$-genus 0 , then it is a rational normal scroll and the ruling of $W$ induces $a$ fibration $V \rightarrow \mathbb{P}^{1}$ whose general fibre is a smooth plane quartic; 
(ii) if $W$ has $\Delta$-genus 1 , then $W$ is normal and $V$ is the intersection of $W$ with a cubic hypersurface. Moreover, in this case the canonical ring of $Y$ is generated in degree 1 , hence the canonical map is a morphism and $V$ is the canonical model of $Y$.

Proof. Cf. [Ko, Theorems 3.1, 4.2, 6.2]. The statement on the normality of $W$ and on the generation of the canonical ring of $Y$ is contained in the proof of Theorem $3.1 \mathrm{of}[\mathrm{Ko}]$ ( $\mathrm{cf}$. 3.6, ibid.).

We will see that in our case $W$ has $\Delta$-genus 1 . In order to prove this we need the following:

Lemma 5.2. The surface $V$ does not have a $G$-invariant free pencil $|F|$ of curves of genus $g(F) \leq 4$.

Proof. Assume that such a pencil $|F|$ exists and denote by $\left|F^{\prime}\right|$ the pencil induced by $|F|$ on $S$.

Let $H$ be the subgroup of $G$ consisting of the elements that act trivially on $|F|$ and let $h$ be the order of $H$. The general $F^{\prime}$ is isomorphic to $F / H$ for some $F$, hence $g\left(F^{\prime}\right)=$ $1+(g(F)-1) / h$ (recall that $H$ acts freely). Since $g(F) \leq 4$, we have either $h=1$ and $g(F)=g\left(F^{\prime}\right)$, or $h=3, g(F)=4, g\left(F^{\prime}\right)=2$.

If $h=1$, then $G=\mathbb{Z}_{9}$, since Aut $|F|=$ Aut $\mathbb{P}^{1}$ does not have a subgroup isomorphic to $\mathbb{Z}_{3}^{2}$. But then $\left|F^{\prime}\right|$ is a pencil of genus $\leq 4$ with two fibres of multiplicity 9 , contradicting the adjunction formula.

If $h=3$, then the pencil $\left|F^{\prime}\right|$ has two triple fibres, corresponding to the two fixed points of the action of $G / H$ on $\mathbb{P}^{1}=|F|$. This again contradicts the adjunction formula, since $g\left(F^{\prime}\right)=2$.

Proposition 5.3. The threefold $W \subset \mathbb{P}^{7}$ has $\Delta$-genus 1 .

Proof. By Proposition 5.1 it is enough to exclude that $W$ is a rational normal scroll. So assume by contradiction that this is the case and denote by $f: V \rightarrow \mathbb{P}^{1}$ the fibration induced by the ruling of $W$. The general fibre $F$ of $f$ is smooth of genus 3 by Proposition 5.1 (i), and by construction the $G$-action on $V$ preserves the fibration $f$. This contradicts Lemma 5.2

Since $W$ has $\Delta$-genus 1 by Proposition 5.3 , it follows by Proposition 5.1 (ii) that the canonical image $V$ of $Y$ is the intersection of $W$ with a cubic hypersurface. As a consequence, we get the following:

Corollary 5.4. The $G$-action on $\mathbb{P}^{7}$ restricts to an action on $W$ which is free outside a finite subset of $W$.

Proof. As already remarked, $G$ acts on $V \subset W \subset \mathbb{P}^{7}$ compatibly. By Propositions 5.1 and 5.3. the surface $V$ is the canonical model of $Y$ and thus $G$ acts freely on $V$. Since by Proposition 5.1.ii) the divisor $V \subset W$ is very ample, it follows that the set of points of $W$ with nontrivial stabilizer has dimension $\leq 0$.

We also need the following two elementary results. 
Lemma 5.5. Assume that $G=\mathbb{Z}_{3}^{2}$ acts on $\mathbb{P}^{2}$ so that the action is free outside a finite set. Then we can choose generators $g_{1}, g_{2} \in G$ and homogeneous coordinates $\left(x_{0}, x_{1}, x_{2}\right)$ on $\mathbb{P}^{2}$ so that the action is as follows:

$$
g_{1}:\left(x_{0}, x_{1}, x_{2}\right) \mapsto\left(x_{0}, \omega x_{1}, \omega^{2} x_{2}\right), \quad g_{2}:\left(x_{0}, x_{1}, x_{2}\right) \mapsto\left(x_{1}, x_{2}, x_{0}\right),
$$

where $\omega \neq 1$ is a third root of 1.

Proof. The fixed locus of a nontrivial element $g_{1} \in G$ consists of three points. In suitable homogeneous coordinates the action of $g_{1}$ is given by

$$
g_{1}:\left(x_{0}, x_{1}, x_{2}\right) \mapsto\left(x_{0}, \omega x_{1}, \omega^{2} x_{2}\right) .
$$

Now consider $g_{2} \in G \backslash\left\langle g_{1}\right\rangle$. The element $g_{2}$ acts on the fixed points of $g_{1}$. If this action is trivial, then $g_{2}$ acts by

$$
g_{2}:\left(x_{0}, x_{1}, x_{2}\right) \mapsto\left(x_{0}, \omega^{a} x_{1}, \omega^{b} x_{2}\right)
$$

for some $a, b \in \mathbb{Z}_{3} \backslash\{0\}$. Then either $g_{1} g_{2}$ or $g_{1} g_{2}^{2}$ fixes a line pointwise, contradicting the assumptions. Hence we conclude that $g_{2}$ permutes the fixed points of $g_{1}$ cyclically. Up to rescaling the coordinates, and possibly replacing $g_{2}$ by $g_{2}^{2}$, the action can be written as stated.

Lemma 5.6. Let $G$ be a group of order 9 that acts on $\mathbb{P}^{1} \times \mathbb{P}^{1} \times \mathbb{P}^{1}$ freely outside a finite subset. If $G$ permutes the three factors of $\mathbb{P}^{1} \times \mathbb{P}^{1} \times \mathbb{P}^{1}$ in a nontrivial way, then $G=\mathbb{Z}_{9}$ and there are affine coordinates $x, y, z$ on the three copies of $\mathbb{P}^{1}$ such that a generator $g$ of $G$ acts by

$$
g:(x, y, z) \mapsto(y, z, \omega x),
$$

where $\omega$ is a primitive third root of 1 .

Proof. The $G$-action permutes the three factors of $\mathbb{P}^{1} \times \mathbb{P}^{1} \times \mathbb{P}^{1}$, hence induces a homomorphism $\psi: G \rightarrow S_{3}$, which is nontrivial by assumption. Thus the image of $\psi$ has order 3 .

Assume that $G=\mathbb{Z}_{3}^{2}$ and let $g \in G$ be such that $\psi(g)=(123)$. Then there are affine coordinates $x, y, z$ on the three copies of $\mathbb{P}^{1}$ such that $g$ acts as follows:

$$
g:(x, y, z) \mapsto(y, z, x)
$$

Hence $g$ fixes pointwise the diagonal $\left\{(P, P, P): P \in \mathbb{P}^{1}\right\}$, contradicting the assumptions.

Thus $G=\mathbb{Z}_{9}$. Let $g \in G$ be an element such that $\psi(g)=(123)$ (notice that $g$ generates $G$ ). Then in suitable coordinates the action of $g$ can be written as claimed. 
The $G$-action on $H^{0}\left(Y, K_{Y}\right)=H^{0}\left(\mathbb{P}^{7}, \mathcal{O}_{\mathbb{P}^{7}}(1)\right)=H^{0}\left(W, \mathcal{O}_{W}(1)\right)$ induces a linearization of the line bundle $\mathcal{O}_{W}(3)$ and therefore a decomposition

$$
H^{0}\left(W, \mathcal{O}_{W}(3)\right)=\bigoplus_{\chi \in G^{*}} T_{\chi},
$$

where $T_{\chi}$ denotes the eigenspace corresponding to the character $\chi$. In particular, $T_{1}$ is the subspace of $G$-invariant vectors. The following remark will be useful in determining the subsystem of $\left|\mathcal{O}_{W}(3)\right|$ which parametrizes the surfaces $V$.

Lemma 5.7. Let $P \in W$ be a point which is fixed by some nontrivial element $g \in G$. If the system $\left|T_{1}\right|$ is free, then $P$ is a base point of $\left|T_{\chi}\right|$ for every $\chi$ such that $\chi(g) \neq 1$.

Proof. Let $\chi \in G^{*}$ be a character such that $\chi(g) \neq 1$. Fix a section $\sigma_{0} \in T_{1}$ such that $\sigma_{0}(P) \neq 0$. For any $\sigma \in\left|T_{\chi}\right|$ consider the rational function $f_{\sigma}:=\sigma / \sigma_{0}$. The function $f_{\sigma}$ is defined at $P$ and

$$
f_{\sigma}(P)=f_{\sigma}(g(P))=\chi\left(g^{-1}\right) f_{\sigma}(P) .
$$

It follows that $f_{\sigma}(P)=0$ and thus also $\sigma(P)=0$.

Varieties of $\Delta$-genus 1 have been classified by Fujita (cf. [Fu1-Fu3]). We recall that by Proposition 5.1 (ii) the threefold $W$ is normal. Here is the list of normal threefolds of $\Delta$-genus 1 in $\mathbb{P}^{7}$ (cf. [Fu3]):

(1) the cone over a (weak) Del Pezzo surface $\Sigma \subset \mathbb{P}^{6}$ of degree 6;

(2) the Segre embedding of $\mathbb{P}^{1} \times \mathbb{P}^{1} \times \mathbb{P}^{1}$ into $\mathbb{P}^{7}$;

(3) the image of the flag variety $\left\{x_{0} y_{0}+x_{1} y_{1}+x_{2} y_{2}=0\right\} \subset \mathbb{P}^{2} \times \mathbb{P}^{2 *}$ under the Segre embedding of $\mathbb{P}^{2} \times \mathbb{P}^{2 *}$.

(4) four singular examples that are not cones. In the notation of [Fu3], these are the cases (vi), (si31i), (si22i), (si211).

To prove Theorem 3.1 we examine cases (1)-(4) separately. We start by showing that case (4) does not occur.

Case (4). If $W$ is of type (vi), (si31i) or (si22i), then by [Fu3], Theorem 2.9] the singular locus of $W$ consists of a line $r$ and, in case (vi), possibly also of an isolated double point. Hence the line $r$ is mapped to itself by the action of $G$ on $\mathbb{P}^{7}$. Then $Z:=V \cap r$ is the intersection of $r$ with a cubic hypersurface and $G$ acts freely on $Z$. This is not possible, since $Z$ is either equal to $r$ or it consists of at most three points.

If $W$ is of type (si211), then by [Fu3] it is contained in the cone $K$ over $M:=$ $\mathbb{P}\left(\mathcal{O}_{\mathbb{P}^{1}}(1) \oplus \mathcal{O}_{\mathbb{P}^{1}}(1) \oplus \mathcal{O}_{\mathbb{P}^{1}}(2)\right)$ embedded in $\mathbb{P}^{6}$ by its tautological system. We denote by $P$ the vertex of $K$. By [Fu3, Theorem 2.9], $P$ is the only isolated double point of $W$, hence $P$ is a fixed point of $G$. Notice, in particular, that $V$ does not contain $P$. Let $\mathbb{P}^{6} \subset \mathbb{P}^{7}$ be the $G$-invariant hyperplane not containing $P$ and let $q: \mathbb{P}^{7} \rightarrow \mathbb{P}^{6}$ be the projection with centre $P$. The threefold $q(W)$ is $G$-invariant and it is isomorphic to $M$. Hence the map $V \rightarrow \mathbb{P}^{1}$ obtained by composing the projection $q$ with the ruling $M \rightarrow \mathbb{P}^{1}$ gives a free pencil of curves $|F|$ which is acted on by $G$. A general $F$ is the intersection in $\mathbb{P}^{3}$ of a 
cubic (given by the cubic equation of $V$ ) and a quadric through $P$ (corresponding to $W$ ) and therefore it has genus 4 . Hence we have a contradiction to Lemma 5.2

Case (1). We show that this case corresponds to surfaces of type B2.

First of all we show that we have $G=\mathbb{Z}_{3}^{2}$ in this case. The vertex $P$ of the cone $W$ is a fixed point of the $G$-action on $\mathbb{P}^{7}$. In particular, $V$ does not contain $P$.

Since the $G$-action on $\mathbb{P}^{7}$ is induced by a linear representation of $G$ on $H^{0}\left(\mathcal{O}_{\mathbb{P}^{7}}(1)\right)=$ $H^{0}\left(Y, K_{Y}\right)$, there exists a $G$-invariant $\mathbb{P}^{6} \subset \mathbb{P}^{7}$ that does not contain $P$. Let $\Sigma:=W \cap \mathbb{P}^{6}$. Then $\Sigma$ is a (weak) Del Pezzo surface of degree 6 preserved by the $G$-action.

The projection from $\mathbb{P}^{7} \rightarrow \mathbb{P}^{6}$ with centre $P$ restricts to a $G$-invariant degree 3 morphism $p: V \rightarrow \Sigma$. If a point $Q \in \Sigma$ is fixed by every element of $G$, then $G$ permutes the points in the fibre $p^{-1}(Q)$, which consists of at most three points. Since $G$ has order 9 , this contradicts the assumption that $G$ acts freely on $V$. In particular, $G$ cannot be cyclic, since every automorphism of a rational surface has at least one fixed point. So $G=\mathbb{Z}_{3}^{2}$.

The same argument shows that the Del Pezzo surface $\Sigma$ is smooth. Indeed, by [Na, Theorem 8], $\Sigma$ is the blowup of $\mathbb{P}^{2}$ along a curvilinear scheme $Z$ of dimension 0 and length 3. If $Z$ is not reduced, then $\Sigma$ is singular and it has precisely one singular point, which is necessarily fixed by every element of $G$.

Arguing as in the proof of Theorem 4.4, one shows that there is a birational morphism $\varepsilon: \Sigma \rightarrow \mathbb{P}^{2}$ such that $\varepsilon$ contracts three disjoint lines of $\Sigma$ to the coordinate points of $\mathbb{P}^{2}$ and the $G$-action on $\Sigma$ descends to a $G$-action on $\mathbb{P}^{2}$. Assume that this last action is not free outside a codimension 2 subset of $\mathbb{P}^{2}$. Then there exists $g \in G, g \neq 0$, that fixes a line $r$ of $\mathbb{P}^{2}$ pointwise. Since $G$ is abelian, we have $g^{\prime}(r)=r$ for every $g^{\prime} \in G$. In particular, $r$ does not contain any of the exceptional points of $\varepsilon^{-1}$, since these are linearly independent and they are permuted cyclically by $G$. Now let $g^{\prime} \in G$ be such that $g, g^{\prime}$ generate $G$ and let $A \in r$ be a point such that $g^{\prime}(A)=A$. Then the point $A$ is fixed by all the elements of $G$ and therefore the point $\varepsilon^{-1}(A) \in \Sigma$ is also fixed by every element of $G$, contradicting the remarks above.

Thus we conclude that $G$ acts freely on $\mathbb{P}^{2}$ outside a finite set and that for a suitable choice of homogeneous coordinates of $\mathbb{P}^{2}$ the $G$-action can be written as in Lemma 5.5.

Hence we may assume that the $G$-action on $\mathbb{P}^{7}$ is the one described in $\$ 3.3$. The point $P$, being invariant for all the group $G$, is a coordinate point of $\mathbb{P}^{7}$. Let $z_{i j}$ denote the only coordinate that does not vanish at $P$, so that the invariant $\mathbb{P}^{6} \subset \mathbb{P}^{7}$ that does not contain $P$ is defined by $z_{i j}=0$. The representation of $G$ on $H^{0}\left(\mathcal{O}_{\mathbb{P}^{6}}(1)\right)$ contains all the nontrivial characters of $G$ except $\chi_{1}^{i} \chi_{2}^{j}$ (see $\$ 3.3$ for the notation). In turn, $H^{0}\left(\mathcal{O}_{\mathbb{P} 6}(1)\right)$ is isomorphic to the subspace $T \subset H^{0}\left(\mathcal{O}_{\mathbb{P}^{2}}(3)\right)$ of cubics vanishing at the coordinate points of $\mathbb{P}^{2}$, and the $G$-action on $T$ induced by this isomorphism differs from the action given in (3.4) by multiplication by a character of $G$. Hence we have $(i, j)=(0,1)$ or $(i, j)=$ $(0,2)$. It follows that, possibly up to replacing $g_{1}$ by $g_{1}^{2}$, the embedding $\Sigma \subset \mathbb{P}^{6} \subset \mathbb{P}^{7}$ is induced by the rational map (3.5. By Proposition 5.1 ii), $V$ is an element of $\left|\mathcal{O}_{W}(3)\right|$ that is $G$-invariant, hence there is a $\chi \in G^{*}$ such that $V \in\left|T_{\chi}\right|$, where $T_{\chi} \subset H^{0}\left(\mathcal{O}_{W}(3)\right)$ is the eigenspace corresponding to $\chi$. We have seen in $\$ 3.3$ that the system $\left|T_{1}\right|$ is free. Hence by Lemma 5.7 the vertex $P$ of $W$ is in the base locus of the system $\left|T_{\chi}\right|$ for every 
$1 \neq \chi \in G^{*}$. Since $G$ acts freely on $V$, it follows that $V$ belongs to $\left|T_{1}\right|$, and the minimal desingularization of $S:=V / G$ is a surface of type $\mathrm{B} 2$.

Case (2). We show that this case corresponds to surfaces of type A. We use the notation of $\S 3$ for the homogeneous and affine coordinates on $W=\mathbb{P}^{1} \times \mathbb{P}^{1} \times \mathbb{P}^{1}$.

For $i=1,2,3$, the projection onto the $i$-th factor $p_{i}: W \rightarrow \mathbb{P}^{1}$ restricts on $V$ to a free pencil of genus 4. By Lemma 5.2, these pencils are not $G$-invariant, hence there is at least one element of $G$ that permutes them. Since $G$ acts freely outside a finite subset, by Lemma 5.6 we have $G=\mathbb{Z}_{9}$ and $G$ acts as in Lemma5.6

By Proposition 5.1(ii), $V$ is an element of $\mid \mathcal{O}_{W}$ (3) $\mid$ that is $G$-invariant, hence there is a $\chi \in G^{*}$ such that $V \in\left|T_{\chi}\right|$, where $T_{\chi} \subset H^{0}\left(\mathcal{O}_{W}(3)\right)$ is the eigenspace corresponding to $\chi$. We have seen in $\$ 3.1$ that the system $\left|T_{1}\right|$ is free. Let $Q \in W$ be the point with affine coordinates $x=y=z=0$. The point $Q$ is fixed by all the group $G$, hence, by Lemma 5.7 $Q$ is in the base locus of the system $\left|T_{\chi}\right|$ for every $1 \neq \chi \in G^{*}$. Since $G$ acts freely on $V$, it follows that $V$ belongs to $\left|T_{1}\right|$, and the minimal desingularization of $S:=V / G$ is a surface of type A.

Case (3). We show that this case corresponds to surfaces of type B1.

Here $W$ is the flag variety $\left\{x_{0} y_{0}+x_{1} y_{1}+x_{2} y_{2}=0\right\} \subset \mathbb{P}^{2} \times \mathbb{P}^{2}$, embedded in $\mathbb{P}^{7}$ as a hyperplane section of the Segre embedding $\mathbb{P}^{2} \times \mathbb{P}^{2^{*}} \hookrightarrow \mathbb{P}^{8}$ (cf. \$3.2). By Lemma 3.7, the action of $G$ on $W$ is induced by a $G$-action on $\mathbb{P}^{2}$. The fixed points on $W$ of an element $g \in$ Aut $\mathbb{P}^{2}$ correspond to pairs $(P, r)$ where $P \in \mathbb{P}^{2}$ is a fixed point of $g, r \in \mathbb{P}^{2^{*}}$ is a fixed line and $P \in r$. Since $G$ acts freely outside a finite subset of $W$, the elements of $G$ have finitely many fixed points on $\mathbb{P}^{2} \times \mathbb{P}^{2 *}$. Hence the $G$-action on $\mathbb{P}^{2}$ is free outside a finite subset of $\mathbb{P}^{2}$. Assume that $G=\mathbb{Z}_{9}$ and let $g \in G$ be a generator. Then there are homogeneous coordinates such that $g$ acts by

$$
\left(x_{0}, x_{1}, x_{2}\right) \mapsto\left(x_{0}, \zeta x_{1}, \zeta^{k} x_{2}\right),
$$

where $\zeta$ is a primitive 9-th root of 1 . Then $x_{0} y_{0}$ and $x_{1} y_{1}$ restrict on $W$ to independent sections of $\mathcal{O}_{W}(1,1)$ that belong to the same $G$-eigenspace. This contradicts Proposition 2.4 Hence $G=\mathbb{Z}_{3}^{2}$ and the action of $G$ on $\mathbb{P}^{2}$ can be written as in Lemma 5.6

By Proposition 5.1 iii), $V$ is an element of $\left|\mathcal{O}_{W}(3,3)\right|$ that is $G$-invariant, hence there is a $\chi \in G^{*}$ such that $V \in\left|T_{\chi}\right|$, where $T_{\chi} \subset H^{0}\left(\mathcal{O}_{W}(3,3)\right)$ is the eigenspace corresponding to $\chi$. We have seen in $\$ 3.2$ that the system $\left|T_{1}\right|$ is free. We observe that for every $0 \neq g \in G$ there is a point $Q \in W$ such that $g Q=Q$. Thus by Lemma5.7, $Q$ is in the base locus of the system $\left|T_{\chi}\right|$ if $\chi(g) \neq 1$. Since $G$ acts freely on $V$, it follows that $V$ belongs to $\left|T_{1}\right|$, and the minimal desingularization of $S:=V / G$ is a surface of type B1.

Acknowledgments. We are grateful to Miles Reid for all his suggestions (mathematical, linguistic, typographical), which improved substantially the presentation of this paper. In particular we wish to thank him for pointing out the unified presentation of surfaces of type B1 and B2 (see 3.4.

We are also indebted to the referee for his thorough reading of the paper and for suggesting further improvements of the presentation.

The first author is a member of the Center for Mathematical Analysis, Geometry and Dynamical Systems and the second author is a member of G.N.S.A.G.A.-I.N.d.A.M. This research was partially supported by the Italian project "Geometria sulle varietà algebriche" (PRIN COFIN 2004) and by FCT (Portugal) through program POCTI/FEDER and Project POCTI/MAT/44068/2002. 


\section{References}

[BPHV] Barth, W., Peters, C., Hulek, K., Van de Ven, A.: Compact Complex Surfaces. 2nd enlarged ed., Ergeb. Math. Grenzgeb. 4, Springer (2004) Zbl 1036.14016 MR 2030225

[Be] Beauville, A.: L'application canonique pour les surfaces de type général. Invent. Math. 55, 121-140 (1979) Zbl 0403.14006 MR 0553705

[CMP] Calabri, A., Mendes Lopes, M., Pardini, R.: Involutions on numerical Campedelli surfaces. Tohoku Math. J. 60, 1-22 (2008)

[Ci] Ciliberto, C.: The bicanonical map for surfaces of general type. In: Algebraic Geometry (Santa Cruz, CA, 1995), Proc. Sympos. Pure Math. 62, Part 1, Amer. Math. Soc., 57-84 (1997) Zbl 0929.14022 MR 1492518

[Fu1] Fujita, T.: Classification of projective varieties of $\Delta$-genus one. Proc. Japan Acad. Ser. A 58, 113-116 (1982) Zbl 0568.14018 MR 0664549

[Fu2] Fujita, T.: On polarized varieties of small $\Delta$-genera. Tohoku Math. J. 34, 319-341 (1982) Zbl 0489.14002 MR 0676113

[Fu3] Fujita, T.: Projective varieties of $\Delta$-genus one. In: Algebraic and Topological Theoriesto the memory of Dr. Takehiko Miyata, Kinokuniya Bookstore, 149-175 (1985) Zbl 0800.14020 MR 1102257

[Ko] Konno, K.: Algebraic surfaces of general type with $c_{1}^{2}=3 p_{g}-6$. Math. Ann. 290, 77-107 (1991) Zbl 0711.14021 MR 1107664

[MP] Mendes Lopes, M., Pardini, R.: On the algebraic fundamental group of surfaces with \begin{tabular}{llll}
$K^{2} \leq 3 \chi$ & . J. Differential Geom. 77, 189-199 (2007) Zbl pre05227609 & MR 2355783 \\
\hline
\end{tabular}

[Na] Nagata, M.: On rational surfaces I. Mem. Coll. Sci. Univ. Kyoto Ser. A 32, 351-370 (1960) Zbl 0100.16703 MR 0126443

[Re1] Reid, M.: Young person's guide to canonical singularities. In: Algebraic Geometry (Bowdoin, 1985), Proc. Sympos. Pure Math. 46, Part 1, Amer. Math. Soc. 345-414 (1987) Zbl 0634.14003 MR 0927963

[Re2] Reid, M.: Surfaces with $p_{g}=0, K_{S}^{2}=2$. http://www.maths.warwick.ac.uk/\%miles/surf/

[Xi1] Xiao, G.: Surfaces fibrées en courbes de genre deux. Lecture Notes in Math. 1137, Springer (1985) Zbl 0579.14028 MR 0872271

[Xi2] Xiao, G.: Finitude de l'application bicanonique des surfaces de type général. Bull. Soc. Math. France 113, 23-51 (1985) Zbl 0611.14031 MR 0807825 